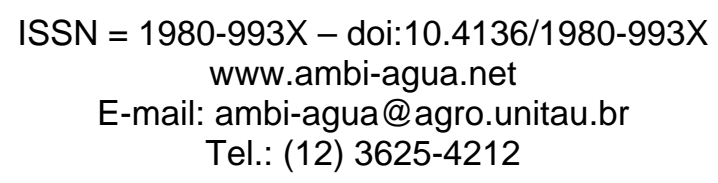

\title{
Parametrização de modelos de mineralização do nitrogênio orgânico em solo tratado com água residuária da suinocultura ${ }^{1}$
}

(doi:10.4136/ambi-agua.140)

\author{
Flávia Mariani Barros²; Mauro Aparecido Martinez³ ${ }^{3}$ Antonio Teixeira de Matos³ ${ }^{3}$ Júlio \\ César Lima Neves ${ }^{4}$; Demétrius David da Silva ${ }^{3}$ \\ ${ }^{1}$ Trabalho extraído da dissertação de mestrado da primeira autora. \\ ${ }^{2}$ Universidade Estadual do Sudoeste da Bahia - UESB \\ E-mail: mariamariani@yahoo.com.br \\ ${ }^{3}$ DEA - Departamento de Engenharia Agrícola - UFV \\ E-mail:mmauro@ufv.br; atmatos@ufv.br; david@ufv.br \\ ${ }^{4}$ DPS - Departamento de Solos - UFV \\ E-mail: julio_n2003@yahoo.com.br
}

\section{RESUMO}

Pouco se sabe a respeito da dinâmica da mineralização do nitrogênio orgânico contido nos dejetos de suínos, tornando-se necessário a adequação de modelos para sua predição. $\mathrm{O}$ objetivo do presente trabalho foi parametrizar e avaliar modelos de mineralização do nitrogênio orgânico em solo tratado com água residuária da suinocultura (ARS), sob diferentes temperaturas e conteúdos de água. Amostras de $57,3 \mathrm{~cm}^{3}$ de Latossolo VermelhoAmarelo distrófico foram misturadas com ARS, numa dose de aplicação de $400 \mathrm{~kg} \mathrm{ha}^{-1}$ de nitrogênio, colocadas em recipientes plásticos e incubadas sob quatro diferentes temperaturas $\left(15,20,25,35^{\circ} \mathrm{C}\right)$ e conteúdos de água correspondentes às tensões de 10, 30, 200 e $1500 \mathrm{kPa}$. Aos 3, 6, 12, 24, 48, 96 dias de incubação, foram retiradas amostras do solo incubado para quantificação das concentrações de amônio e nitrato. A partir dos valores de nitrogênio orgânico mineralizado, obtidos ao longo de diferentes tempos de incubação, foram determinados os parâmetros dos modelos de mineralização do nitrogênio no solo. O valor da concentração de nitrogênio potencialmente mineralizável $\left(\mathrm{N}_{0}\right)$ em solo com aplicação de ARS foi 2,5 vezes superior em relação à concentração obtida em solo sem aplicação de ARS. A constante de mineralização (k) em solo com ARS foi sempre maior em relação ao solo sem ARS. Houve tendência do modelo exponencial simples subestimar os valores da concentração de nitrogênio mineralizado. Na maioria das situações, o modelo potencial foi mais eficiente que o modelo exponencial simples para predizer a mineralização do nitrogênio orgânico.

Palavras-chave: dejeto de suínos; disposição de residuos; nitrato; amônio.

\section{Parametrization of organic nitrogen mineralization models in soil treated with swine raising wastewater}

\begin{abstract}
Little is known about the mineralization dynamic of organic nitrogen contained in swine manure, so models need to be adjusted for its prediction. The objective of the present study was to parameterize and assess models of organic nitrogen mineralization in soil treated with swine raising wastewater (SRW) at different temperatures and water contents. Samples of $57.3 \mathrm{~cm}^{3}$ of dystrophic Red-Yellow Latosol were mixed with SRW at the application dose of $400 \mathrm{~kg} \mathrm{ha}^{-1}$ nitrogen, placed in plastic cups and incubated at four different temperatures (15, 20,25 and $35^{\circ} \mathrm{C}$ ) and water contents corresponding to tensions of 10, 30, 200 and $1500 \mathrm{kPa}$. Samples were removed from the incubated soil after 3, 6, 12, 24, 48 and 96 days to quantify the ammonium and nitrate concentrations. The parameters of the soil organic nitrogen
\end{abstract}


BARRoS, F. M.; MARTINEZ, M. A.; MATOS, A. T.; NEVES, J. C. L.; SILVA, D. D. Parametrização de modelos de mineralização do nitrogênio orgânico em solo tratado com água residuária da suinocultura. AmbiAgua, Taubaté, v. 5, n. 2, p. 99-111, 2010. (doi:10.4136/ambi-agua.140)

mineralization models were determined from the organic nitrogen mineralization values obtained over the different incubation periods. The value of the potentially mineralizable nitrogen $\left(\mathrm{N}_{0}\right)$ in soil with application of SRW was superior that of the soil without application of SRW. The mineralization constant $(\mathrm{k})$ in soil with application of SRW was always superior that of the soil without application of SRW. There was a tendency for the simple exponential model to underestimate the values of the mineralized nitrogen concentration. In most of the situations the potential model was more efficient than the simple exponential model to predict the mineralization of the organic nitrogen.

Keywords: swine dejects; residue disposal; nitrate; ammonium.

\section{INTRODUÇÃO}

O nitrogênio ocupa posição de destaque entre os elementos essenciais ao crescimento e desenvolvimento das plantas, sendo um dos macronutrientes mais caros e instáveis no solo e, por isso, é considerado, juntamente com o fósforo, o principal fator limitante à produção agrícola em solos de regiões de clima tropical (Matos, 2007).

Nos países tropicais, a limitada disponibilidade de nitrogênio nos solos e os altos custos dos fertilizantes nitrogenados têm levado à utilização de resíduos animais, tais como dejeto de suínos, como fonte de nutrientes (Vanlauwe et al., 1997). Dessa maneira, a utilização adequada desses dejetos, além de contribuir para o aumento da fertilidade do solo, também pode ser uma solução para diminuição da degradação ambiental causada pelo lançamento destes diretamente nos cursos de água (Souza et al., 2009).

Os critérios que predizem a liberação de nitrogênio dos resíduos com base em suas características químicas podem facilitar a seleção e o manejo desses materiais.

A baixa eficiência do uso de nitrogênio proveniente dos resíduos orgânicos é atribuída à falta de sincronismo entre a sua liberação e a demanda pelas plantas, associada às perdas por lixiviação (Myers et al., 1994). A sincronia não necessariamente aumenta os rendimentos das plantas em curto prazo, mas pode reduzir as perdas de nitrogênio e melhorar a fertilidade do solo em longo prazo.

O conhecimento das características do dejeto de suínos relacionados à degradação microbiológica do nitrogênio orgânico pode contribuir para a previsão de seu comportamento no solo, permitindo definir parâmetros úteis ao estabelecimento das doses máximas a serem aplicadas aos solos, em função do nitrogênio disponibilizado às plantas.

A concentração de nitrogênio mineralizado em função do tempo pode ser estimada com a utilização de modelos matemáticos. Diversos modelos foram elaborados para estimar a mineralização do nitrogênio orgânico nos solos, tais como, o modelo exponencial simples proposto por Stanford e Smith (1972), o modelo potencial proposto por Broadbent e Nakashima (1968) e o modelo proposto por Marion et al. (1981).

Avaliando o potencial de mineralização de nitrogênio orgânico, Stanford e Smith (1972) verificaram que as constantes de mineralização estimadas por meio do modelo exponencial simples foram semelhantes para diferentes solos dos Estados Unidos.

Em estudos realizados por Camargo et al. (2002), avaliando vários modelos empíricos para a predição da mineralização do nitrogênio orgânico em solos do Rio Grande do Sul, o melhor ajuste foi obtido com o modelo exponencial simples, proposto por Stanford e Smith (1972).

Em razão dos inúmeros fatores que interferem na mineralização do nitrogênio, tem sido difícil encontrar características do solo que indiretamente possibilitem as estimativas dos parâmetros dos modelos de mineralização do nitrogênio. Dentre os vários fatores que interferem nessa mineralização, a temperatura e o conteúdo de água do solo são os que necessitam ser mais estudados do ponto de vista da modelagem do processo. 
BARRoS, F. M.; MARTINEZ, M. A.; MATOS, A. T.; NEVES, J. C. L.; SILVA, D. D. Parametrização de modelos de mineralização do nitrogênio orgânico em solo tratado com água residuária da suinocultura. AmbiAgua, Taubaté, v. 5, n. 2, p. 99-111, 2010. (doi:10.4136/ambi-agua.140)

Nesse contexto, objetivou-se, com este trabalho, parametrizar e avaliar os modelos de mineralização do nitrogênio orgânico propostos por Broadbent e Nakashima (1968) e Stanford e Smith (1972) em solo tratado com água residuária da suinocultura (ARS), sob diferentes condições de temperatura e conteúdo de água.

\section{MATERIAL E MÉTODOS}

O trabalho foi conduzido nos Laboratórios de Água e Solo e de Qualidade da Água do Departamento de Engenharia Agrícola da Universidade Federal de Viçosa (UFV), no período de maio a agosto de 2004.

Amostras deformadas de 57,3 $\mathrm{cm}^{3}$ de Latossolo Vermelho-Amarelo distrófico de textura muito argilosa foram misturadas com uma quantidade de ARS correspondente à aplicação de $400 \mathrm{~kg} \mathrm{ha}^{-1}$ de nitrogênio, considerando uma camada de solo de $20 \mathrm{~cm}$ de profundidade (Westerman et al., 1987). Essas foram, então, colocadas em recipientes plásticos com volume de $60 \mathrm{~cm}^{3}$ e incubadas sob quatro diferentes temperaturas $\left(15,20,25\right.$ e $\left.35^{\circ} \mathrm{C}\right)$, em câmera BOD com variação de $\pm 1^{\circ} \mathrm{C}$ e quatro conteúdos de água distintos (correspondentes às tensões de 10, 30, 200 e $1500 \mathrm{kPa}$ ). O conteúdo de água no solo incubado foi monitorado por pesagem, sendo ajustado diariamente com a adição de água deionizada.

As amostras de solo tratado com a ARS foram retiradas aos 3, 6, 12, 24, 48 e 96 dias de incubação, para a quantificação das concentrações de amônio e de nitrato. Para a determinação da forma amônio, foi utilizado o método descrito por Kempers e Zweers (1986). As análises de nitrato foram realizadas segundo o método descrito por Yang et al. (1998), fundamentadas no método colorimétrico. O amônio e o nitrato das amostras de solo foram extraídos utilizando-se a solução de cloreto de potássio $1 \mathrm{~mol} \mathrm{~L}^{-1}$. O nitrogênio mineralizado foi obtido por meio da soma do nitrato com o amônio. As mesmas análises descritas anteriormente foram também realizadas em solo que não recebeu ARS.

Com base nos valores de nitrogênio mineralizado, obtidos ao longo de diferentes tempos de incubação, foram determinados os parâmetros do modelo proposto por Broadbent e Nakashima (1968) (Equação 1) para cada combinação de temperatura e conteúdo de água no solo. Com relação aos parâmetros da equação proposta por Stanford e Smith (1972) (Equação 2), o $\mathrm{N}_{0}$ foi ajustado para a combinação ótima entre temperatura e conteúdo de água, e os valores da constante de mineralização foram estimados para cada combinação de temperatura e conteúdo de água. As regressões não lineares foram ajustadas pelo método quasi-Newton do programa STATISTICA, versão 6.0 (Statsoft, 1996), em nível de observação.

$$
\begin{gathered}
N_{m}=A t^{b} \\
N_{m}=N_{0}\left(1-e^{-k t}\right)
\end{gathered}
$$

em que:

$\mathrm{N}_{\mathrm{m}}=$ concentração de nitrogênio orgânico mineralizado em determinado tempo (t)

$(\mathrm{mg} \mathrm{kg})^{-1}$;

$\mathrm{A}=$ Taxa de mineralização ( $\left.\mathrm{mg} \mathrm{kg}^{-1} \mathrm{dia}^{-\mathrm{b}}\right)$;

$\mathrm{b}=$ constante;

$\mathrm{t}$ = tempo decorrido após o início da incubação (dia).

$\mathrm{N}_{0}$ = concentração de nitrogênio potencialmente mineralizável no solo ( $\mathrm{mg} \mathrm{kg}^{-1}$ );

$\mathrm{k}=$ constante de mineralização $\left(\operatorname{dia}^{-1}\right)$.

O delineamento experimental constituiu-se de blocos casualizados com quatro repetições, nos quais foi utilizado esquema fatorial $4 \times 4$ (4 conteúdos de água e 4 
BARros, F. M.; MARTINEZ, M. A.; MATOS, A. T.; NEVES, J. C. L.; SILVA, D. D. Parametrização de modelos de mineralização do nitrogênio orgânico em solo tratado com água residuária da suinocultura. AmbiAgua, Taubaté, v. 5, n. 2, p. 99-111, 2010. (doi:10.4136/ambi-agua.140)

temperaturas) em parcelas subdivididas, as quais foram avaliadas ao longo do tempo de incubação.

Para cada modelo ajustado, calculou-se o coeficiente residual de massa (CRM) e a eficiência do modelo (EF), que foram utilizados para avaliar a precisão do modelo (Equações 3 e 4 , respectivamente).

$$
\begin{gathered}
\text { CRM }=\left[\sum_{i=1}^{n} P_{i}-\sum_{i=1}^{n} O_{i}\right] / \sum_{i=1}^{n} O_{i} \\
E F=\left[\sum_{i=1}^{n}\left(O_{i}-\bar{O}\right)^{2}-\sum_{i=1}^{n}\left(P_{i}-O_{i}\right)^{2}\right] / \sum_{i=1}^{n}\left(O_{i}-\bar{O}\right)^{2}
\end{gathered}
$$

em que:

$\mathrm{P}_{\mathrm{i}}=$ Valores estimados pelo modelo;

$\mathrm{O}_{\mathrm{i}}=$ Valores observados experimentalmente;

$\bar{O}=$ Média dos valores observados;

n = número de observações.

O CRM representa uma medida da tendência do modelo em superestimar ou subestimar os valores observados, sendo o valor zero indicativo de condição ótima (Loague e Green, 1991; Xevi et al., 1996). Valores positivos indicam tendência de superestimação do modelo enquanto valores negativos indicam tendência de subestimação do modelo (Loague e Green, 1991; Xevi et al., 1996).

A eficiência do modelo (EF) apresenta valores menores ou iguais a 1, com o valor 1 representando ajuste perfeito (Loague e Green, 1991; Xevi, 1996).

\section{RESULTADOS E DISCUSSÃO}

\subsection{Modelo exponencial simples}

Nas Tabelas 1 e 2 estão apresentados os valores dos parâmetros $\mathrm{N}_{0}$ e k do modelo exponencial simples proposto por Stanford e Smith (1972), em solo sem e com a aplicação de ARS, respectivamente.

O valor do $\mathrm{N}_{0}$ em solo com aplicação de ARS (207,16 $\mathrm{mg} \mathrm{kg}^{-1}$ ) foi superior ao do solo sem aplicação de ARS (81,76 mg kg$\left.{ }^{-1}\right)$. O aumento do nitrogênio está associado à matéria orgânica aportada ao solo pela ARS.

O valor médio de $\mathrm{N}_{0}$ encontrado por Parentoni et al. (1988), para diferentes tipos de solos do Rio Grande do Sul, incubados a $24^{\circ} \mathrm{C}$, por 210 dias foi de $31 \mathrm{mg} \mathrm{kg}^{-1}$, sendo este valor menor que o valor encontrado no presente experimento para solo sem aplicação de ARS $\left(81,76 \mathrm{mg} \mathrm{kg}^{-1}\right)$. Esse fato pode ter ocorrido devido ao menor tempo de incubação deste estudo quando comparado ao estudo realizado por Parentoni et al. (1988) e/ou devido às diferenças nas características de cada tipo de solo.

Em trabalho realizado por Boeira et al. (2002), com o objetivo de quantificar, em laboratório, o potencial de mineralização do nitrogênio orgânico em Latossolo tratado com diferentes doses e tipos de lodo de esgoto, incubados durante 105 dias, sob temperatura de $24^{\circ} \mathrm{C}$ e conteúdo de água mantido na capacidade de campo do solo, foi verificada uma variação do $\mathrm{N}_{0}$ de 43,9 a 131,0 $\mathrm{mg} \mathrm{kg}^{-1}$, para o lodo da Estação de Tratamento de Franca e de 44,6 a 264,6 mg kg-1 para o lodo da Estação de Tratamento de Barueri, dependendo da dose aplicada. A dose aplicada de $429 \mathrm{mg} \mathrm{kg}^{-1}$ de nitrogênio ao solo (lodo da Estação de Tratamento de Esgoto de Barueri) foi a que conduziu a valores de $\mathrm{N}_{0}\left(174,8 \mathrm{mg} \mathrm{kg}^{-1}\right)$ mais próximos aos obtidos no presente trabalho quando da aplicação de ARS. Deve-se ressaltar, 
BARROS, F. M.; MARTINEZ, M. A.; MATOS, A. T.; NEVES, J. C. L.; SILVA, D. D. Parametrização de modelos de mineralização do nitrogênio orgânico em solo tratado com água residuária da suinocultura. AmbiAgua, Taubaté, v. 5, n. 2, p. 99-111, 2010. (doi:10.4136/ambi-agua.140)

porém, que a ARS e o lodo de esgoto apresentam características diferentes, tais como quantidade de nitrogênio e matéria orgânica, entre outros.

Tabela 1. Valores dos parâmetros do modelo exponencial simples proposto por Stanford e Smith (1972) ajustados ao nitrogênio mineralizado, em solo sem ARS incubado sob diferentes temperaturas e conteúdos de água.

\begin{tabular}{|c|c|c|c|c|c|c|}
\hline \multicolumn{2}{|c|}{ Tratamentos } & \multicolumn{2}{|c|}{$N_{m}=N_{0}\left(1-e^{-k t}\right)$} & \multirow[b]{2}{*}{$\mathbf{R}^{2(6)}$} & \multirow[b]{2}{*}{$\mathbf{C R M}^{(3)}$} & \multirow[b]{2}{*}{$\mathbf{E F}^{(4)}$} \\
\hline $\begin{array}{l}\text { Temperatura } \\
\quad\left({ }^{\circ} \mathrm{C}\right)\end{array}$ & $\begin{array}{c}\text { Conteúdo de água } \\
\left(\mathrm{kg} \mathrm{kg}^{-1}\right)\end{array}$ & $\begin{array}{c}\mathrm{N}_{\mathbf{0}}^{\left({ }^{(1)}\right.} \\
\left(\mathrm{mg} \mathrm{kg}^{-1}\right)\end{array}$ & $\begin{array}{c}\mathbf{k}^{(2)} \\
\left(\mathbf{d i a}^{-1}\right)\end{array}$ & & & \\
\hline 15 & $\begin{array}{l}0,300(10 \mathrm{kPa}) \\
0,281(30 \mathrm{kPa}) \\
0,241(200 \mathrm{kPa}) \\
0,218(1500 \mathrm{kPa})\end{array}$ & 81,76 & $\begin{array}{l}0,0109^{* *} \\
0,0098^{*} \\
0,0096^{\circ} \\
0,0079^{\circ}\end{array}$ & $\begin{array}{l}0,01 \\
0,01 \\
0,01 \\
0,22\end{array}$ & $\begin{array}{l}-0,268 \\
-0,275 \\
-0,228 \\
-0,228 \\
\end{array}$ & $\begin{array}{l}\mathrm{NE}^{(5)} \\
\mathrm{NE} \\
\mathrm{NE} \\
0,212\end{array}$ \\
\hline 20 & $\begin{array}{l}0,300(10 \mathrm{kPa}) \\
0,281(30 \mathrm{kPa}) \\
0,241(200 \mathrm{kPa}) \\
0,218(1500 \mathrm{kPa})\end{array}$ & 81,76 & $\begin{array}{l}0,0100^{* *} \\
0,0104^{* *} \\
0,0094^{*} \\
0,0077^{\text {ns }}\end{array}$ & $\begin{array}{l}0,06 \\
0,30 \\
0,01 \\
0,01\end{array}$ & $\begin{array}{l}-0,225 \\
-0,217 \\
-0,212 \\
-0,256\end{array}$ & $\begin{array}{l}0,057 \\
0,277 \\
\mathrm{NE} \\
\mathrm{NE}\end{array}$ \\
\hline 25 & $\begin{array}{l}0,300(10 \mathrm{kPa}) \\
0,281(30 \mathrm{kPa}) \\
0,241(200 \mathrm{kPa}) \\
0,218(1500 \mathrm{kPa})\end{array}$ & 81,76 & $\begin{array}{l}0,0201^{* *} \\
0,0183^{* *} \\
0,0157^{* *} \\
0,0094^{* *}\end{array}$ & $\begin{array}{l}0,91 \\
0,93 \\
0,92 \\
0,92\end{array}$ & $\begin{array}{l}-0,123 \\
-0,125 \\
-0,137 \\
-0,204\end{array}$ & $\begin{array}{l}0,908 \\
0,922 \\
0,917 \\
0,918\end{array}$ \\
\hline 35 & $\begin{array}{l}0,300(10 \mathrm{kPa}) \\
0,281(30 \mathrm{kPa}) \\
0,241(200 \mathrm{kPa}) \\
0,218(1500 \mathrm{kPa})\end{array}$ & 81,76 & $\begin{array}{l}0,0282^{*} \\
0,0269^{*} \\
0,0221^{* *} \\
0,0170^{\circ}\end{array}$ & $\begin{array}{l}0,85 \\
0,85 \\
0,80 \\
0,82\end{array}$ & $\begin{array}{l}-0,108 \\
-0,112 \\
-0,141 \\
-0,138\end{array}$ & $\begin{array}{l}0,840 \\
0,847 \\
0,782 \\
0,823\end{array}$ \\
\hline
\end{tabular}

ns, $0, *, * *$, não significativo, significativo a $10 \%, 5 \%$ e $1 \%$ respectivamente, pelo teste $t ;{ }^{(1)}$ Concentração de nitrogênio orgânico potencialmente mineralizável; ${ }^{(2)}$ Constante de mineralização; ${ }^{(3)}$ Coeficiente Residual de Massa; ${ }^{(4)}$ Eficiência do modelo; ${ }^{(5)}$ Eficiência menor que 0,01; ${ }^{(6)}$ Porcentagem de variância explicada.

Na Figura 1 está apresentado o comportamento do nitrogênio mineralizado em função do tempo para solos com e sem ARS nas temperaturas de 15 e $35^{\circ} \mathrm{C}$ e conteúdo de água de $0,300 \mathrm{~kg} \mathrm{~kg}^{-1}$.

Conforme pode-se observar na Figura 1 e nas Tabelas 1 e 2, o modelo proposto por Stanford e Smith (1972) foi, em geral, mais eficiente para predição do nitrogênio mineralizado em temperaturas mais altas. Isso pode ser devido ao fato de que, em temperaturas mais altas (até determinada faixa de valores), a mineralização do nitrogênio se processa de forma mais rápida, e o contrário ocorre em temperaturas mais baixas. Dessa forma, as estimativas dessa variável são mais precisas quando a mineralização se processa de forma mais rápida, já que o período de incubação foi de 96 dias. Já em temperaturas mais baixas, deve haver um maior tempo de incubação para se obter estimativas mais precisas do nitrogênio mineralizado.

Parentoni et al. (1988) observaram que o aumento do tempo de incubação melhora as estimativas do $\mathrm{N}_{0}$. Para Litossolos e Cambissolos, três semanas de incubação foram suficientes para estimar $\mathrm{N}_{0}$. Para os Podzólicos, foram necessários 42 dias de incubação. Já para os Hidromórficos, 70 dias de incubação foram suficientes. Os Latossolos, dentre os grupos estudados, foram os que exigiram mais tempo, com cerca de 210 dias de incubação, tempo este superior ao utilizado no presente trabalho. 
BARROS, F. M.; MARTINEZ, M. A.; MATOS, A. T.; NEVES, J. C. L.; SILVA, D. D. Parametrização de modelos de mineralização do nitrogênio orgânico em solo tratado com água residuária da suinocultura. AmbiAgua, Taubaté, v. 5, n. 2, p. 99-111, 2010. (doi:10.4136/ambi-agua.140)

Tabela 2. Valores dos parâmetros do modelo exponencial simples proposto por Stanford e Smith (1972) ajustados ao nitrogênio mineralizado, em solo com ARS incubado sob diferentes temperaturas e conteúdos de água.

\begin{tabular}{|c|c|c|c|c|c|c|}
\hline \multicolumn{2}{|c|}{ Tratamentos } & \multicolumn{2}{|c|}{$N_{m}=N_{0}\left(1-e^{-k t}\right)$} & \multirow[b]{2}{*}{$\mathbf{R}^{2(6)}$} & \multirow[b]{2}{*}{$\mathrm{CRM}^{(3)}$} & \multirow[b]{2}{*}{$E F^{(4)}$} \\
\hline $\begin{array}{c}\text { Temperatura } \\
\left({ }^{\circ} \mathrm{C}\right)\end{array}$ & $\begin{array}{c}\text { Conteúdo de água } \\
\left(\mathrm{kg} \mathrm{kg}^{-1}\right)\end{array}$ & $\begin{array}{c}\mathrm{N}_{0}^{(1)} \\
\left(\mathrm{mg} \mathrm{kg}^{-1}\right)\end{array}$ & $\begin{array}{c}\mathbf{k}^{(2)} \\
\left(\operatorname{dia}^{-1}\right)\end{array}$ & & & \\
\hline 15 & $\begin{array}{l}0,300(10 \mathrm{kPa}) \\
0,281(30 \mathrm{kPa}) \\
0,241(200 \mathrm{kPa}) \\
0,218(1500 \mathrm{kPa})\end{array}$ & 207,16 & $\begin{array}{l}0,0312 * * \\
0,0261^{* *} \\
0,0145^{*} \\
0,0107^{*}\end{array}$ & $\begin{array}{l}0,14 \\
0,18 \\
0,01 \\
0,01\end{array}$ & $\begin{array}{l}-0,0652 \\
-0,0949 \\
-0,2332 \\
-0,3140\end{array}$ & $\begin{array}{l}0,137 \\
0,174 \\
\mathrm{NE}^{(5)} \\
\mathrm{NE}\end{array}$ \\
\hline 20 & $\begin{array}{l}0,300(10 \mathrm{kPa}) \\
0,281(30 \mathrm{kPa}) \\
0,241(200 \mathrm{kPa}) \\
0,218(1500 \mathrm{kPa})\end{array}$ & 207,16 & $\begin{array}{l}0,0508 * * \\
0,0348 * * \\
0,0289 * * \\
0,0086 * *\end{array}$ & $\begin{array}{l}0,81 \\
0,59 \\
0,04 \\
0,01\end{array}$ & $\begin{array}{r}-0,0270 \\
0,1067 \\
-0,0441 \\
-0,3300 \\
\end{array}$ & $\begin{array}{l}0,805 \\
0,584 \\
0,037 \\
\mathrm{NE}\end{array}$ \\
\hline 25 & $\begin{array}{l}0,300(10 \mathrm{kPa}) \\
0,281(30 \mathrm{kPa}) \\
0,241(200 \mathrm{kPa}) \\
0,218(1500 \mathrm{kPa})\end{array}$ & 207,16 & $\begin{array}{l}0,0679 * * \\
0,0611^{* *} \\
0,0347 * * \\
0,0118 * *\end{array}$ & $\begin{array}{l}0,77 \\
0,75 \\
0,37 \\
0,01\end{array}$ & $\begin{array}{r}0,0032 \\
-0,4943 \\
-0,0409 \\
-0,2789\end{array}$ & $\begin{array}{l}0,771 \\
0,749 \\
0,367 \\
\mathrm{NE}\end{array}$ \\
\hline 35 & $\begin{array}{l}0,300(10 \mathrm{kPa}) \\
0,281(30 \mathrm{kPa}) \\
0,241(200 \mathrm{kPa}) \\
0,218(1500 \mathrm{kPa})\end{array}$ & 207,16 & $\begin{array}{l}0,0956 * * \\
0,0742 * * \\
0,0545 * * \\
0,0367 * *\end{array}$ & $\begin{array}{l}0,95 \\
0,84 \\
0,69 \\
0,71\end{array}$ & $\begin{array}{r}-0,0030 \\
0,0365 \\
0,0346 \\
-0,0129\end{array}$ & $\begin{array}{l}0,945 \\
0,840 \\
0,689 \\
0,708\end{array}$ \\
\hline
\end{tabular}

*, **, significativo a 5\% e 1\% respectivamente, pelo teste t; ${ }^{(1)}$ Concentração de nitrogênio orgânico potencialmente mineralizável; ${ }^{(2)}$ Constante de mineralização; ${ }^{(3)}$ Coeficiente Residual de Massa; ${ }^{(4)}$ Eficiência do modelo; ${ }^{(5)}$ Eficiência menor que 0,$01 ;{ }^{(6)}$ Porcentagem de variância explicada.

Quanto mais intemperizado o solo, maior o tempo de incubação que ele necessita para melhores estimativas do $\mathrm{N}_{0}$, o que pode ser devido a maior quantidade de óxidos de ferro e alumínio e maior grau de recalcitrância nesses solos, enquanto que em solos menos intemperizados há maior quantidade de nutrientes disponíveis.

Os valores da constante de mineralização $(\mathrm{k})$ variaram no solo sem ARS de 0,0077 a 0,0282 $\mathrm{dia}^{-1}$ (Tabela 1), e em solo com ARS, de 0,0086 a 0,0956 dia ${ }^{-1}$ (Tabela 2), indicando, assim, grande diversidade no valor desse parâmetro, assim como relatado por Parentoni et al. (1988).

Nota-se que, em geral, houve aumento da constante de mineralização (Tabelas 1 e 2 ) com o aumento da temperatura e do conteúdo de água, tanto em solo com aplicação de ARS quanto em solo sem aplicação de ARS, demonstrando que maiores temperaturas e conteúdos de água (dentro de determinada faixa de valores) favorecem o processo de mineralização do nitrogênio.

Pode-se observar graficamente (Figura 1) que, quanto maior a constante de mineralização maior foi o nitrogênio mineralizado nos primeiros dias de incubação e mais rápido ocorreu a estabilização dessas concentrações.

Em solo sem ARS, a correlação entre a constante de mineralização foi muito mais alta em relação à temperatura $(\mathrm{r}=0,85 ; \mathrm{p}<0,01)$ que em relação ao conteúdo de água $(\mathrm{r}=0,38$; $\mathrm{p}<0,01)$. Já em solo com ARS, a correlação entre a constate de mineralização e a temperatura $(\mathrm{r}=0,68 ; \mathrm{p}<0,01)$ e entre a constante e o conteúdo de água $(\mathrm{r}=0,687 ; \mathrm{p}<0,01)$ foram próximas. 
BARros, F. M.; MARTINEZ, M. A.; MATOS, A. T.; NEVES, J. C. L.; SILVA, D. D. Parametrização de modelos de mineralização do nitrogênio orgânico em solo tratado com água residuária da suinocultura. AmbiAgua, Taubaté, v. 5, n. 2, p. 99-111, 2010. (doi:10.4136/ambi-agua.140)

(a)

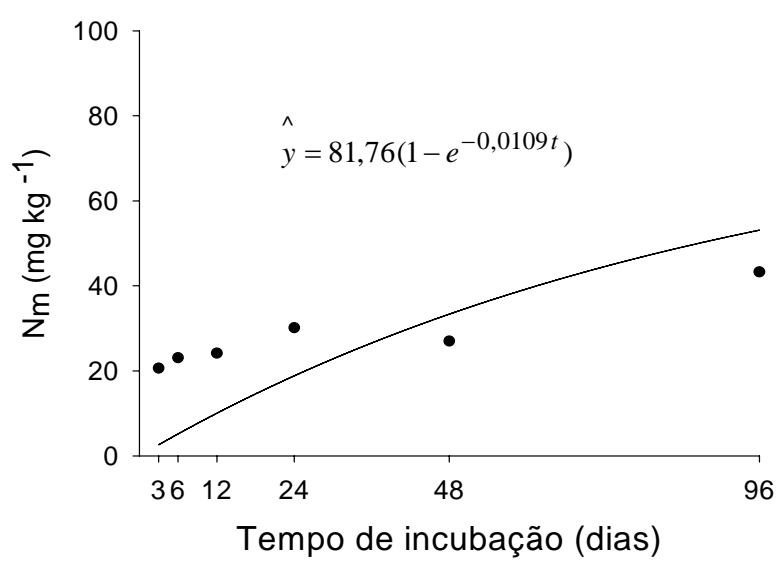

(c)

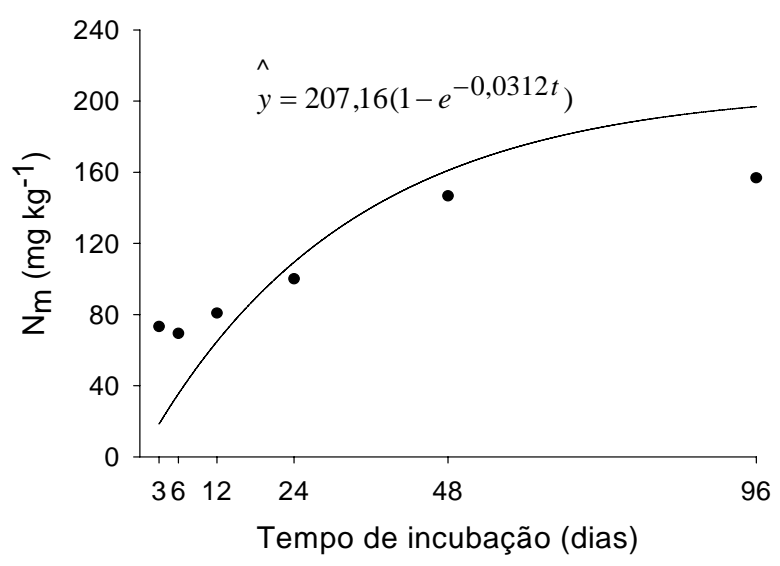

(b)

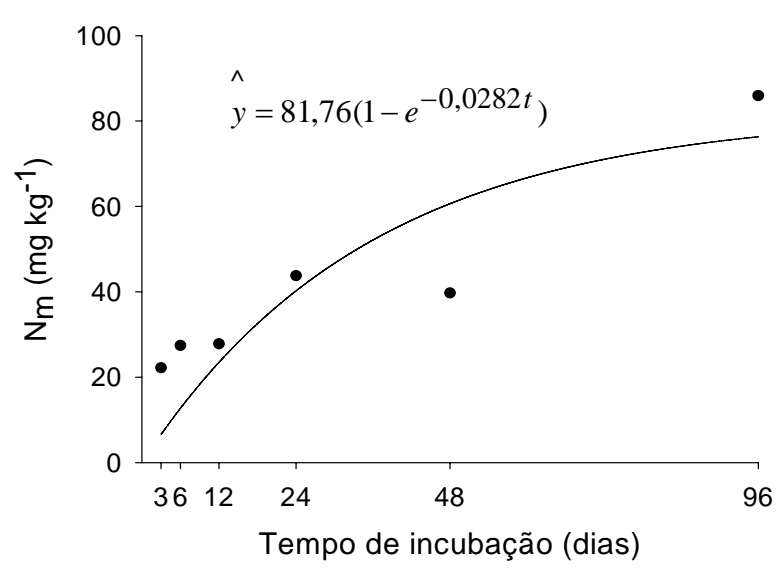

(d)

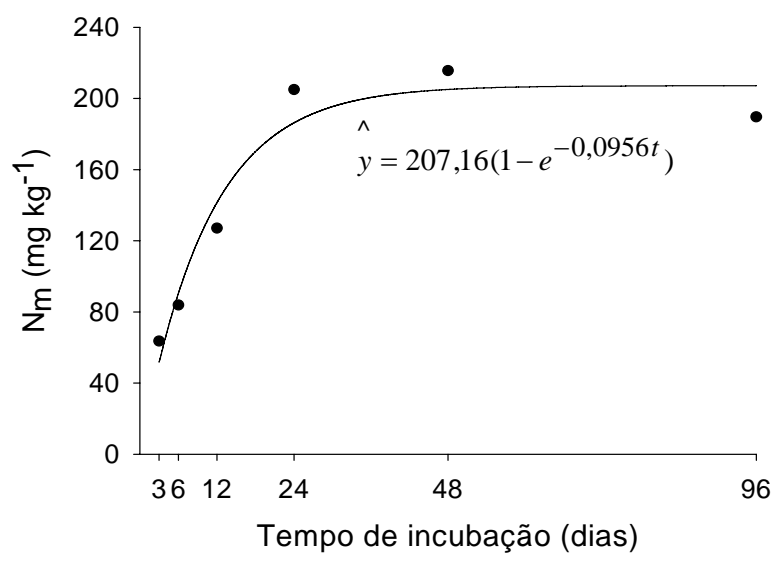

Figura 1. Nitrogênio mineralizado $\left(\mathrm{N}_{\mathrm{m}}\right)$ em função do tempo incubação $(\mathrm{t})$, sob temperatura de $15^{\circ} \mathrm{C}$ e conteúdo de água de $0,300 \mathrm{~kg} \mathrm{~kg}^{-1}$, em solo sem ARS (a) e em solo com ARS (c) e sob temperatura de $35^{\circ} \mathrm{C}$ e conteúdo de água de $0,300 \mathrm{~kg} \mathrm{~kg}^{-1}$, em solo sem ARS (b) e em solo com ARS (d).

Na condição do presente trabalho, que mais se assemelha à do experimento realizado por Boeira et al. (2002) (no qual foi utilizado solo tratado com lodo de esgoto), isto é, solo sob temperatura de $25^{\circ} \mathrm{C}$ e conteúdo de água de $0,281 \mathrm{~kg} \mathrm{~kg}^{-1}$, o valor de $\mathrm{k}$ foi de $0,0611 \mathrm{dia}^{-1}$ para solo com ARS, sendo esse valor superior ao encontrado por Boeira et al. (2002), que foi de $0,0075 \mathrm{dia}^{-1}$. O maior valor de $\mathrm{k}$ no presente trabalho indica mais rápido fornecimento de nitrogênio mineral à solução do solo pela ARS quando comparado ao solo em que foi aplicado lodo de esgoto. Isso pode ser devido à maior recalcitrância do material orgânico contido no lodo de esgoto em comparação com a ARS.

Os valores encontrados para a constante $\mathrm{k}$ em diferentes tipos de solo do Rio Grande do Sul, incubados a $24^{\circ} \mathrm{C}$, durante 210 dias, variaram entre 0,0005 e 0,0172 dia ${ }^{-1}$ (Parentoni et al.,1988). Salcedo et al. (1985), trabalhando com um Podzólico Vermelho Amarelolatossólico arenoso, representativo dos tabuleiros costeiros de Pernambuco, obtiveram um valor de $\mathrm{k}$ de $0,011 \mathrm{dia}^{-1}$, com solo incubado a $35^{\circ} \mathrm{C}$, enquanto Alves et al. (1999) encontraram valores de $\mathrm{k}$ entre 0,0185 e 0,0757 $\mathrm{dia}^{-1}$ para solos da região de Pernambuco, nas mesmas condições experimentais, sendo os valores encontrados superiores aos obtidos no presente experimento. 
BARRoS, F. M.; MARTINEZ, M. A.; MATOS, A. T.; NEVES, J. C. L.; SILVA, D. D. Parametrização de modelos de mineralização do nitrogênio orgânico em solo tratado com água residuária da suinocultura. AmbiAgua, Taubaté, v. 5, n. 2, p. 99-111, 2010. (doi:10.4136/ambi-agua.140)

Santos (2001) verificou que as constantes de mineralização para solos brasileiros variaram de 0,0035 a $0,0276 \mathrm{dia}^{-1}$, sendo superiores às constantes obtidas para solos dos Estados Unidos, observando uma variação que sugere maior labilidade da matéria orgânica ou maior atividade microbiana nos solos tropicais. Os valores da constante $\mathrm{k}$ encontrados por Santos (2001) foram próximos aos valores encontrados no presente trabalho em solo sem aplicação de ARS $\left(0,0077\right.$ a 0,0282 dia $\left.^{-1}\right)$.

Camargo et al. (2002) relataram em seu trabalho, realizado com 30 solos do Rio Grande do Sul, que as taxas de mineralização encontradas foram mais altas que as citadas por Stanford e Smith (1972) em solos dos Estados Unidos, o que, segundo o mesmo autor, pode ser devido à natureza do processo, ocorrendo mais rápida mineralização nas primeiras semanas, seguidas por período relativamente longo de estabilização, corroborando com os resultados obtidos no presente trabalho. A ocorrência dessas altas taxas pode estar associada ao aumento da atividade microbiana, induzida pela manipulação e preparo das amostras (Stanford e Smith, 1972; Camargo et al., 2002).

Kliemann (1973), trabalhando com vinte solos do Rio Grande do Sul, verificou que a maior parte do nitrogênio total presente estava na forma de aminoácidos $(42,4 \%)$ e que essa fração foi a que mais contribuiu para o fornecimento de nitrogênio às plantas.

Tanto em solo sem ARS como em solo com ARS, o Coeficiente Residual de Massa $(\mathrm{CRM})$ foi negativo em todos os tratamentos (Tabelas 1 e 2), exceto para a temperatura de $20^{\circ} \mathrm{C}$ e conteúdo de água de $0,218 \mathrm{~kg} \mathrm{~kg}^{-1}$, temperatura de $25^{\circ} \mathrm{C}$ e conteúdo de água de $0,300 \mathrm{~kg} \mathrm{~kg}^{-1}$ e temperatura de $35^{\circ} \mathrm{C}$ e conteúdos de água de 0,281 e $0,241 \mathrm{~kg} \mathrm{~kg}^{-1}$, todos em solo com presença de ARS. Isso indica a tendência do modelo exponencial simples, na maioria dos casos, subestimar os valores de nitrogênio mineralizado.

Segundo Cardoso et al. (1992), o solo se constitui de inúmeros microssítios, caracterizados não apenas pelas condições edafoclimáticas, mas ainda por características peculiares, e tais características podem variar muito dentro de um mesmo local, pois se lida com grande número de micro-habitats microbianos que diferem entre si. Logo, a grande variação que ocorreu na mineralização do nitrogênio orgânico pode estar ligada a outros fatores, além da temperatura e do conteúdo de água no meio como, por exemplo, às características do solo, à qualidade da ARS adicionada ao solo, à variação na população de micro-organismos, entre outros.

\subsection{Modelo potencial}

Nas Tabelas 3 e 4 estão apresentados os parâmetros referentes ao modelo potencial, em solo sem aplicação de ARS e em solo com aplicação de ARS, respectivamente. Pode-se notar que os valores da taxa de mineralização (A) são sempre maiores em solo com aplicação de ARS em relação ao solo sem aplicação da ARS.

A taxa de mineralização em solo sem aplicação de ARS não se correlacionou com a temperatura, porém, correlacionou-se positivamente com o conteúdo de água do solo ( $\mathrm{r}=0,70$; $\mathrm{p}<0,01)$. Já em solo com aplicação de ARS, a taxa de mineralização não se correlacionou nem com a temperatura nem com o conteúdo de água no solo.

O parâmetro b do modelo potencial, em solo sem ARS, correlacionou-se positivamente com a temperatura $(r=0,78 ; \mathrm{p}<0,01)$, enquanto que em solo com ARS, o parâmetro correlacionou-se positivamente tanto com a temperatura $(\mathrm{r}=0,35 ; \mathrm{p}<0,1)$ quanto com $\mathrm{o}$ conteúdo de água $(\mathrm{r}=0,60 ; \mathrm{p}<0,01)$.

Em solos sem aplicação de ARS (Tabela 3), houve tendência do modelo em superestimar a concentração de nitrogênio mineralizado nos tratamentos submetidos à temperatura de $15^{\circ} \mathrm{C}$ e conteúdos de água de 0,300 e $0,281 \mathrm{~kg} \mathrm{~kg}^{-1}$ e, também, sob a temperatura de $20^{\circ} \mathrm{C}$ e conteúdo de água de $0,300 \mathrm{~kg} \mathrm{~kg}^{-1}$ (CRM positivo). Nos outros tratamentos, os valores de 
BARRoS, F. M.; MARTINEZ, M. A.; MATOS, A. T.; NEVES, J. C. L.; SILVA, D. D. Parametrização de modelos de mineralização do nitrogênio orgânico em solo tratado com água residuária da suinocultura. AmbiAgua, Taubaté, v. 5, n. 2, p. 99-111, 2010. (doi:10.4136/ambi-agua.140)

CRM foram negativos, indicando tendência do modelo em subestimar os valores da concentração de nitrogênio mineralizado.

Tabela 3. Valores dos parâmetros do modelo potencial proposto por Broadbent e Nakashima (1968) ajustado ao nitrogênio mineralizado, em solo sem ARS incubado sob diferentes temperaturas e conteúdos de água.

\begin{tabular}{|c|c|c|c|c|c|c|}
\hline \multicolumn{2}{|c|}{ Tratamentos } & \multicolumn{2}{|c|}{$\mathbf{N}_{\mathrm{m}}=A \mathbf{t}^{\mathrm{b}}$} & \multirow[b]{2}{*}{ 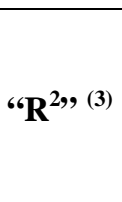 } & \multirow[b]{2}{*}{$\mathbf{C R M}^{(4)}$} & \multirow[b]{2}{*}{$\mathbf{E F}^{(5)}$} \\
\hline $\begin{array}{c}\text { Temperatur } \\
\text { a } \\
\left({ }^{\circ} \mathrm{C}\right)\end{array}$ & $\begin{array}{l}\text { Conteúdo de } \\
\text { água } \\
\left(\mathrm{kg} \mathrm{kg}^{-1}\right)\end{array}$ & $\begin{array}{c}\mathrm{A}^{(1)} \\
(\mathrm{mg} \mathrm{kg} \mathrm{dia-b})\end{array}$ & $\mathbf{b}^{(2)}$ & & & \\
\hline 15 & $\begin{array}{l}0,300(10 \mathrm{kPa}) \\
0,281(30 \mathrm{kPa}) \\
0,241(200 \mathrm{kPa}) \\
0,218(1500 \mathrm{kPa})\end{array}$ & $\begin{array}{l}15,13^{* *} \\
13,37^{* *} \\
8,67^{* *} \\
6,49^{* *}\end{array}$ & $\begin{array}{l}0,2069^{* *} \\
0,2243^{* *} \\
0,3321^{* *} \\
0,3726 * *\end{array}$ & $\begin{array}{l}0,77 \\
0,63 \\
0,78 \\
0,80\end{array}$ & $\begin{array}{r}0,006 \\
0,013 \\
-0,007 \\
-0,001\end{array}$ & $\begin{array}{l}0,790 \\
0,704 \\
0,835 \\
0,806\end{array}$ \\
\hline 20 & $\begin{array}{l}0,300(10 \mathrm{kPa}) \\
0,281(30 \mathrm{kPa}) \\
0,241(200 \mathrm{kPa}) \\
0,218(1500 \mathrm{kPa})\end{array}$ & $\begin{array}{l}9,25^{* *} \\
8,90^{* *} \\
7,28^{* *} \\
8,14^{* *}\end{array}$ & $\begin{array}{l}0,3225^{* *} \\
0,3384 * * \\
0,3744 * * \\
0,3123 * *\end{array}$ & $\begin{array}{l}0,89 \\
0,86 \\
0,87 \\
0,74\end{array}$ & $\begin{array}{r}0,004 \\
-0,007 \\
-0,010 \\
-0,009\end{array}$ & $\begin{array}{l}0,913 \\
0,862 \\
0,872 \\
0,750\end{array}$ \\
\hline 25 & $\begin{array}{l}0,300(10 \mathrm{kPa}) \\
0,281(30 \mathrm{kPa}) \\
0,241(200 \mathrm{kPa}) \\
0,218(1500 \mathrm{kPa})\end{array}$ & $\begin{array}{l}9,85^{* *} \\
7,75^{* *} \\
5,67 * \\
5,64 * *\end{array}$ & $\begin{array}{l}0,4040^{* *} \\
0,4567^{* *} \\
0,5201^{* *} \\
0,4411^{* *}\end{array}$ & $\begin{array}{l}0,94 \\
0,92 \\
0,86 \\
0,69\end{array}$ & $\begin{array}{l}-0,009 \\
-0,015 \\
-0,026 \\
-0,025\end{array}$ & $\begin{array}{l}0,937 \\
0,920 \\
0,860 \\
0,698\end{array}$ \\
\hline 35 & $\begin{array}{l}0,300(10 \mathrm{kPa}) \\
0,281(30 \mathrm{kPa}) \\
0,241(200 \mathrm{kPa}) \\
0,218(1500 \mathrm{kPa})\end{array}$ & $\begin{array}{l}9,91^{* *} \\
10,40^{* *} \\
6,00^{* *} \\
7,08^{* *}\end{array}$ & $\begin{array}{l}0,4487 * * \\
0,4293 * * \\
0,5596 * * \\
0,4732 * *\end{array}$ & $\begin{array}{l}0,84 \\
0,84 \\
0,74 \\
0,66\end{array}$ & $\begin{array}{l}-0,015 \\
-0,014 \\
-0,038 \\
-0,020\end{array}$ & $\begin{array}{l}0,841 \\
0,847 \\
0,750 \\
0,670\end{array}$ \\
\hline
\end{tabular}

*, **, significativo a $5 \%$ e $1 \%$, respectivamente, pelo teste t; ${ }^{(1)}$ Taxa de mineralização ${ }^{(2)}$ Expoente do tempo; ${ }^{(3)}$ Porcentagem da variância explicada; ${ }^{(4)}$ Coeficiente Residual de Massa; ${ }^{(5)}$ Eficiência do modelo.

Em solo com aplicação de ARS (Tabela 4), foi observada tendência do modelo em subestimar a concentração do nitrogênio mineralizado sob a temperatura de $15^{\circ} \mathrm{C}$, exceto para o conteúdo de água de $0,218 \mathrm{~kg} \mathrm{~kg}^{-1}$, em que os resultados estimados pelo modelo ficaram mais próximos dos dados observados, com valor de CRM zero, situação que também ocorreu para os tratamentos com temperatura de $20^{\circ} \mathrm{C}$ e conteúdos de água de 0,300, 0,241 e $0,218 \mathrm{~kg} \mathrm{~kg}^{-1}$ e temperatura de $25^{\circ}$ e conteúdo de água de $0,218 \mathrm{~kg} \mathrm{~kg}^{-1}$. Nos outros tratamentos, como o CRM foi positivo, houve tendência do modelo em superestimar os dados. A magnitude do CRM foi menor em solo com ARS comparado ao solo sem ARS.

Na Figura 2 está apresentado o comportamento do nitrogênio mineralizado em função do tempo para solos com e sem ARS nas temperaturas de 15 e $35^{\circ} \mathrm{C}$ e conteúdo de água de $0,300 \mathrm{~kg} \mathrm{~kg}^{-1}$.

De acordo com a Figura 2, pode-se notar que o modelo potencial descreveu mais precisamente o comportamento do nitrogênio mineralizado em solos com e sem aplicação de ARS na temperatura de $15^{\circ} \mathrm{C}$ quando comparado ao modelo exponencial simples.

\subsection{Eficiência dos modelos estudados}

Considerando os valores da eficiência do modelo, verifica-se que tanto no solo sem (Tabela 3) quanto no solo com aplicação de ARS (Tabela 4), o modelo proposto por Broadbent e Nakashima (1968), em geral, mostrou-se mais eficiente que o modelo proposto por Stanford e Smith (1972) (Tabelas 1 e 2). Exceção deve ser feita ao solo sem ARS na temperatura de $25^{\circ} \mathrm{C}$ e conteúdos de água de 0,$281 ; 0,241$ e 0,218 e temperatura de $35^{\circ} \mathrm{C}$ e conteúdos de água de 0,241 e 0,218 e para o solo com ARS na temperatura de $35^{\circ} \mathrm{C}$ e 
BARROS, F. M.; MARTINEZ, M. A.; MATOS, A. T.; NEVES, J. C. L.; SILVA, D. D. Parametrização de modelos de mineralização do nitrogênio orgânico em solo tratado com água residuária da suinocultura. AmbiAgua, Taubaté, v. 5, n. 2, p. 99-111, 2010. (doi:10.4136/ambi-agua.140)

conteúdos de água de 0,300 e $0,281 \mathrm{~kg} \mathrm{~kg}^{-1} 35^{\circ} \mathrm{C}$. Alcântra et al. (2007) observaram, em experimento com lodo de curtume, que o modelo de Stanford e Smith não se ajustou adequadamente (lodo do decantador primário), devido às diferentes frações com variados graus de recalcitrância

Pode-se notar que, no modelo potencial, houve tendência da diminuição da eficiência do modelo com a diminuição do conteúdo de água sob temperaturas de 15,20 e $25^{\circ} \mathrm{C}$, em solo com ARS, enquanto que na temperatura de $35^{\circ} \mathrm{C}$, essa tendência foi contrária. $\mathrm{O}$ mesmo ocorreu para solos sem ARS nas temperaturas de 20 , 25 e $35^{\circ} \mathrm{C}$, com tendência contrária na temperatura de $15^{\circ} \mathrm{C}$.

Tabela 4. Valores dos parâmetros do modelo potencial proposto por Broadbent e Nakashima (1968) ajustado ao nitrogênio mineralizado, em solo com ARS incubado sob diferentes temperaturas e conteúdos de água.

\begin{tabular}{|c|c|c|c|c|c|c|}
\hline \multicolumn{2}{|c|}{ Tratamentos } & \multicolumn{2}{|c|}{$\mathbf{N}_{\mathrm{m}}=\mathbf{A t ^ { b }}$} & \multirow[b]{2}{*}{ “ $R^{2 »,(3)}$} & \multirow[b]{2}{*}{$\mathrm{CRM}^{(4)}$} & \multirow[b]{2}{*}{$\mathbf{E F}^{(5)}$} \\
\hline $\begin{array}{c}\text { Temperatur } \\
\text { a } \\
\left({ }^{\circ} \mathrm{C}\right)\end{array}$ & $\begin{array}{c}\text { Conteúdo de } \\
\text { água } \\
\left(\mathrm{kg} \mathrm{kg}^{-1}\right)\end{array}$ & $\begin{array}{c}\mathrm{A}^{(1)} \\
\left(\mathrm{mg} \mathrm{kg} \mathrm{dia}{ }^{-b}\right)\end{array}$ & $\mathbf{b}^{(2)}$ & & & \\
\hline 15 & $\begin{array}{l}0,300(10 \mathrm{kPa}) \\
0,281(30 \mathrm{kPa}) \\
0,241(200 \mathrm{kPa}) \\
0,218(1500 \mathrm{kPa})\end{array}$ & $\begin{array}{l}44,84^{* *} \\
39,90^{* *} \\
48,66^{* *} \\
56,36^{* *}\end{array}$ & $\begin{array}{l}0,2792 * * \\
0,2975 * * \\
0,1787 * * \\
0,0983 * *\end{array}$ & $\begin{array}{l}0,92 \\
0,93 \\
0,86 \\
0,60\end{array}$ & $\begin{array}{r}-0,001 \\
-0,002 \\
-0,001 \\
0,000\end{array}$ & $\begin{array}{l}0,924 \\
0,937 \\
0,870 \\
0,628\end{array}$ \\
\hline 20 & $\begin{array}{l}0,300(10 \mathrm{kPa}) \\
0,281(30 \mathrm{kPa}) \\
0,241(200 \mathrm{kPa}) \\
0,218(1500 \mathrm{kPa})\end{array}$ & $\begin{array}{l}42,72 * * \\
39,61 * * \\
43,65 * * \\
48,44 * *\end{array}$ & $\begin{array}{l}0,3452 * * \\
0,3292 * * \\
0,2696 * * \\
0,1072 * *\end{array}$ & $\begin{array}{l}0,88 \\
0,78 \\
0,67 \\
0,28\end{array}$ & $\begin{array}{l}0,000 \\
0,001 \\
0,000 \\
0,000\end{array}$ & $\begin{array}{l}0,886 \\
0,791 \\
0,673 \\
0,284\end{array}$ \\
\hline 25 & $\begin{array}{l}0,300(10 \mathrm{kPa}) \\
0,281(30 \mathrm{kPa}) \\
0,241(200 \mathrm{kPa}) \\
0,218(1500 \mathrm{kPa})\end{array}$ & $\begin{array}{l}48,32 * * \\
45,29 * * \\
42,68 * * \\
50,65 * *\end{array}$ & $\begin{array}{l}0,3298 * * \\
0,3382 * * \\
0,3028 * * \\
0,1410 * *\end{array}$ & $\begin{array}{r}0,82 \\
0,85 \\
0,76 \\
0,435\end{array}$ & $\begin{array}{l}0,002 \\
0,002 \\
0,002 \\
0,000\end{array}$ & $\begin{array}{l}0,823 \\
0,853 \\
0,764 \\
0,441\end{array}$ \\
\hline 35 & $\begin{array}{l}0,300(10 \mathrm{kPa}) \\
0,281(30 \mathrm{kPa}) \\
0,241(200 \mathrm{kPa}) \\
0,218(1500 \mathrm{kPa})\end{array}$ & $\begin{array}{l}65,21 * * \\
58,80 * * \\
48,41 * * \\
35,64 * *\end{array}$ & $\begin{array}{l}0,2727 * * \\
0,2694 * * \\
0,2972 * * \\
0,3569 * *\end{array}$ & $\begin{array}{l}0,73 \\
0,69 \\
0,86 \\
0,90\end{array}$ & $\begin{array}{l}0,007 \\
0,007 \\
0,004 \\
0,003\end{array}$ & $\begin{array}{l}0,730 \\
0,692 \\
0,860 \\
0,899\end{array}$ \\
\hline
\end{tabular}

ns, *, **, não significativo, significativo a $5 \%$ e $1 \%$ respectivamente, pelo teste t; ${ }^{(1)}$ Taxa de mineralização; ${ }^{(2)}$ Expoente do tempo; ${ }^{(3)}$ Porcentagem da variância explicada; ${ }^{(4)}$ Coeficiente Residual de Massa; ${ }^{(5)}$ Eficiência do modelo.

Como se pode verificar nas Tabelas 1 e 2, em geral, o modelo proposto por Stanford e Smith (1972), em relação aos diferentes tratamentos, foi mais eficiente para predizer a mineralização do nitrogênio orgânico nas maiores temperaturas $\left(25\right.$ e $\left.35^{\circ} \mathrm{C}\right)$.

O modelo proposto por Stanford e Smith (1972), sendo um modelo de cinética de primeira ordem, é uma função exponencial negativa, isto é, os valores da concentração de nitrogênio mineralizado aumentam exponencialmente no período de tempo inicial e depois tendem a ficar constantes com o passar do tempo. Já no modelo proposto por Broadbent e Nakashima (1968), os valores da concentração de nitrogênio mineralizado não aumentam tanto no início do processo, ficando mais próximos da realidade dos dados observados neste experimento, em temperaturas menores $\left(15\right.$ e $\left.20^{\circ} \mathrm{C}\right)$. 
BARROS, F. M.; MARTINEZ, M. A.; MATOS, A. T.; NEVES, J. C. L.; SILVA, D. D. Parametrização de modelos de mineralização do nitrogênio orgânico em solo tratado com água residuária da suinocultura. AmbiAgua, Taubaté, v. 5, n. 2, p. 99-111, 2010. (doi:10.4136/ambi-agua.140)

(a)

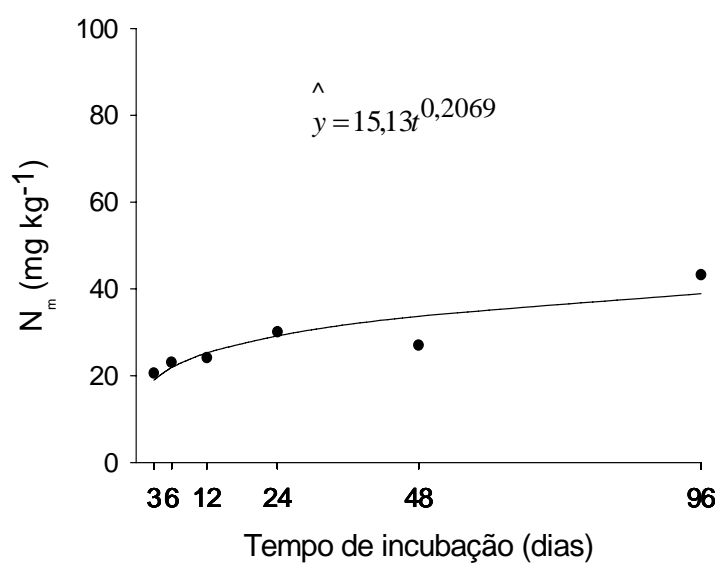

(c)

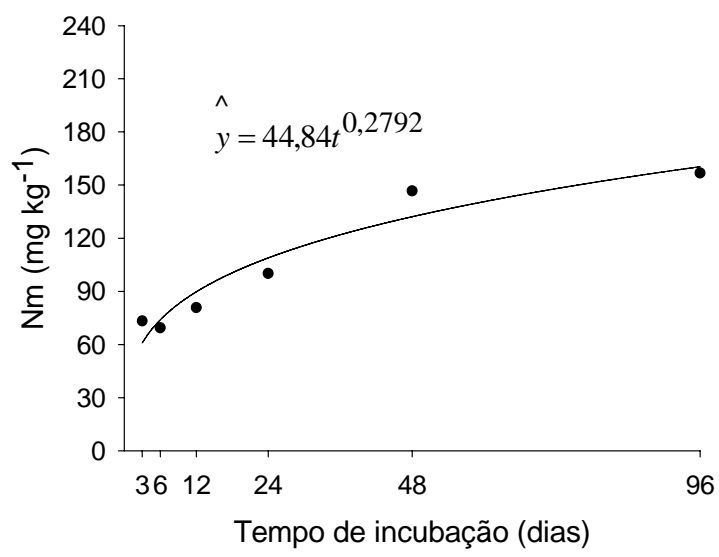

(b)

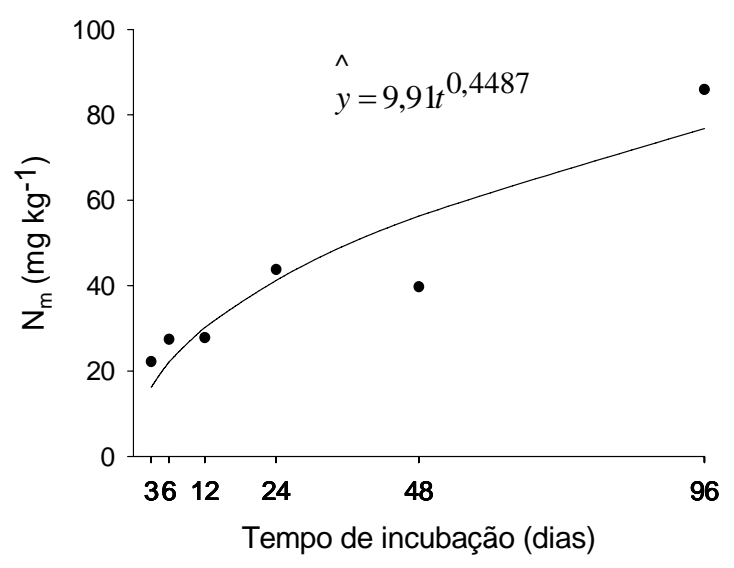

(d)

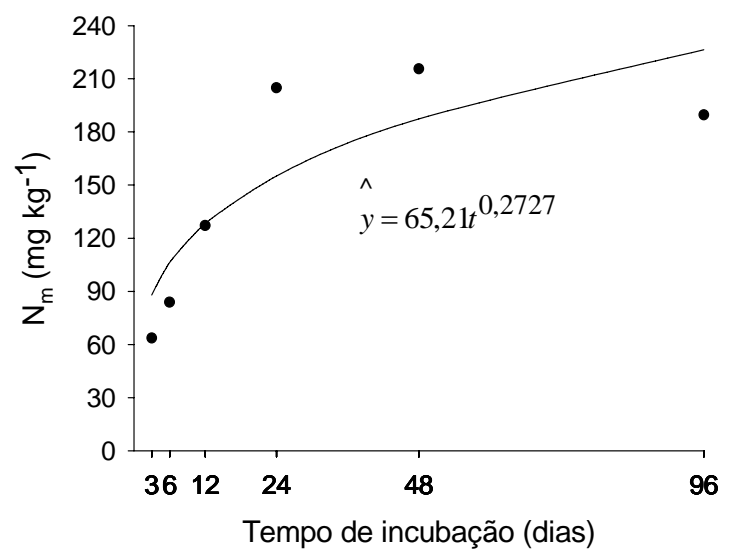

Figura 2. Nitrogênio mineralizado $\left(\mathrm{N}_{\mathrm{m}}\right)$ em função do tempo incubação $(\mathrm{t})$, sob temperatura de $15^{\circ} \mathrm{C}$ e conteúdo de água de $0,300 \mathrm{~kg} \mathrm{~kg}^{-1}$, em solo sem ARS (a) e em solo com ARS (c) e sob temperatura de $35^{\circ} \mathrm{C}$ e conteúdo de água de $0,300 \mathrm{~kg} \mathrm{~kg}^{-1}$, em solo sem ARS (b) e em solo com ARS (d).

Stanford e Smith (1972), trabalhando com mineralização do nitrogênio orgânico, observaram que o modelo proposto por Broadbent e Nakashima (1968) se ajustou bem a maioria dos resultados experimentais. Todavia, eles não deram muita atenção a esse modelo, usando-o apenas para pré-estimar o valor de $\mathrm{N}_{0}$ no modelo exponencial simples (Broadbent, 1986). De fato, o modelo proposto por Broadbent e Nakashima (1968) ajusta-se melhor aos dados que o modelo exponencial simples para alguns solos, não para todos (Broadbent, 1986). Pereira (2003) também verificou bons ajustes aos dados, utilizando o modelo proposto por Broadbent e Nakashima (1968).

\section{CONCLUSÕES}

Tendo por base os resultados obtidos e considerando-se as condições em que o estudo foi realizado, conclui-se que:

- O valor da concentração de nitrogênio potencialmente mineralizável $\left(\mathrm{N}_{0}\right)$ em solo com aplicação de ARS foi 2,5 vezes superior em relação à concentração obtida em solo sem aplicação de ARS;

- A constante de mineralização (k) em solo com ARS foi sempre maior em relação ao solo sem ARS; 
- Houve tendência do modelo exponencial simples em subestimar os valores da concentração de nitrogênio mineralizado, tanto em solo com aplicação de ARS quanto em solo sem aplicação de ARS;

- O modelo exponencial simples foi mais eficiente para predição do nitrogênio mineralizado em maiores temperaturas $\left(25\right.$ e $\left.35^{\circ} \mathrm{C}\right)$ em comparação com as menores temperaturas;

- Na maioria das situações, a eficiência do modelo potencial foi maior que o modelo exponencial simples em relação à predição da mineralização do nitrogênio orgânico.

\section{AGRADECIMENTOS}

Agradecemos à FAPEMIG e ao CNPq pelo apoio concedido ao trabalho de pesquisa.

\section{REFERÊNCIAS}

ALCÂNTRA, M. A. K.; AQUINO NETO, V.; CAMARGO, O. A.; CANTARELLA, H. Mineralização de nitrogênio em solos tratados com lodo de curtume. Pesq. Agrop. Bras., Brasília, v. 42, n. 4, p. 547-555, 2007.

ALVES, G. D.; SAMPAIO, E. V. S. B.; SALCEDO, I. M.; SILVA, V. M. Potencial de mineralização de N em vinte solos de Pernambuco. R. Bras. Ci. Solo, Viçosa, v. 23, p. 245-256, 1999.

BOEIRA, R. C.; LIGO, M. A. V.; DYNIA, J. F. Mineralização de nitrogênio em solo tropical tratado com lodos de esgoto. Pesq. Agrop. Bras, Brasília, v. 37, p. 1639-1647, 2002.

BROADBENT, F. E. Empirical modeling of soil nitrogen mineralization. Soil Sci., Philadelphia, v. 141, p. 208-213, 1986.

BROADBENT, F. E.; NAKASHIMA, T. Plant uptake and residual value of six tagged nitrogen fertilizers. Soil Sci. Soc. Am. Proc., Madison, v. 32, p. 388-392, 1968.

CAMARGO, F. A. O.; GIANELLO, C.; TEDESCO, M. J.; RIBOLD, J.; MEURER, E. J.; BISSANI, C. A. Empirical models to predict soil nitrogen mineralization. Ciência Rural, Santa Maria, v. 32, p. 393-399, 2002.

CARDOSO, J. B. N.; TSAI, S. M.; NEVES, M. C. P. Microbiologia do solo. Campinas: Sociedade Brasileira de Ciência do Solo, 1992. 360 p.

KEMPERS, A. J.; ZWEERS, A. Ammonium determination in soil extracts by the salicylate method. Soil Sci. Plant Anal., Madison, v. 17, p. 715-723, 1986.

KLIEMANN, H. J. Componentes nitrogenados de alguns solos do Rio Grande do Sul e sua relação com a disponibilidade de nitrogênio para as plantas. 1973. $75 f$. Dissertação (Mestrado em Agronomia) -Universidade Federal do Rio Grande do Sul, Porto Alegre, 1973.

LOAGUE, K.; GREEN, R. E. Statistical and graphical methods for evaluating solute transport models: overview and application. J. Contam. Hydrol., Amsterdam, v. 7, p. 51-73, 1991.

MARION, G. M.; KUMMEROW, J.; MILLER, P. C. Predicting nitrogen mineralization in chaparral soils. Soil Sci. Soc. Am. J., Madison, v. 45, p. 956-961, 1981. 
BARROS, F. M.; MARTINEZ, M. A.; MATOS, A. T.; NEVES, J. C. L.; SILVA, D. D. Parametrização de modelos de mineralização do nitrogênio orgânico em solo tratado com água residuária da suinocultura. AmbiAgua, Taubaté, v. 5, n. 2, p. 99-111, 2010. (doi:10.4136/ambi-agua.140)

MATOS, A. T. Disposição de águas residuárias no solo. Viçosa, MG: AEAGRI, 2007. 142p. (Caderno didático, 38).

MYERS, R. J. K.; PALM, C. A.; CUEVAS, E.; GUNATILLE K. E.; BROSSARD, I. U. N. The synchronisations of mineralisation and plant nutrient demand. In: WOOMER, P. L.; SWIFT, M. J. (Eds.). The biological management of tropical soil fertility. Chichester:: John Wiley and Sons, 1994. p. 81-116.

PARENTONI, S. N.; FRANÇA, G. E.; BAHIA FILHO, A. F. C. Avaliação dos conceitos de quantidade e intensidade de mineralização de nitrogênio para trinta solos do Rio Grande do Sul. R. Bras. Ci. Solo, Viçosa, v. 12, p. 225-229, 1988.

PEREIRA, J. M. Predição do índice de mineralização do nitrogênio em solos através da comparação de modelos não lineares. In: SIMPÓSIO DE ESTATÍSTICA APLICADA À EXPERIMENTAÇÃO AGRONÔMICA SEAGRO, 10., Lavras, 2003. Anais... Lavras: UFSCar 2003.

SALCEDO, I. H.; SAMPAIO, E. V. S. B.; ALVES, G. D. Mineralização do carbono e do nitrogênio em solo cultivado com cana de açucar. R. Bras. Ci. Solo, Viçosa, v. 9, p. 3338, 1985.

SANTOS, C. A. A. DOS. Potencial de mineralização e disponibilidade de nitrogênio de solos. 2001. 59f. Dissertação (Mestrado em Ciência do Solo) - Universidade Federal de Viçosa, Viçosa, MG, 2001.

SOUZA, J. A. R.; MOREIRAM D. A.; FERREIRA, P. A.; MATOS, A. T. Variação de nitrogênio e fósforo em solo fertirrigado com efluente do tratamento primário da água residuária da suinocultura. Revista Ambiente \& Água, Taubaté, v. 4, n. 3, 2009.

STANFORD, G.; SMITH, S. J. Nitrogen mineralization potential of soil. Soil Sci. Soc. Am. J., Madison, v. 36, p. 465-471, 1972.

STATSOFT. Statistica for Windows: v. 6.0. Tulsa: Statsoft, 1996.

VANLAUWE, J. D.; SANGINGA, N.; MERCKX, R. Residue quality and decomposition: an unsteady relationship? In: CADISCH, G.; GILLER, K. E. Driven by nature- pant letter quality and decompositions. Wallingford: CAB International, 1997. p. 157- 166.

WESTERMAN, P. W.; KING, L. D.; BURNS, J. C.; CUMMINGS, G. A.; OVERCASH, M. R. Swine manure and lagoon effluent applied to a temperate forage mixture: II Rainfall runoff and soil chemical properties. J. Environ. Qual., v. 16, p. 106-112, 1987.

XEVI, E.; GILLEY, J.; FEYEN, J. Comparative study of two crop yield simulation models. Agric. Water Manage, v. 30, p. 155-173, 1996.

YANG, J. E.; SKOGLEY, E. O.; SCHAFF, B. E.; KIM, J. J. A simple spectrophotometric determination of nitrate in water, resin and soil extracts. Soil Sci. Soc. Sm. J., Madison, v. 62, p. 1108-1115, 1998. 